\title{
On Probability Distribution Solutions of a Functional Equation
}

by

\section{Janusz MORAWIEC and Ludwig REICH}

Presented by Andrzej LASOTA

Summary. Let $0<\beta<\alpha<1$ and let $p \in(0,1)$. We consider the functional equation

$$
\varphi(x)=p \varphi\left(\frac{x-\beta}{1-\beta}\right)+(1-p) \varphi\left(\min \left\{\frac{x}{\alpha}, \frac{x(\alpha-\beta)+\beta(1-\alpha)}{\alpha(1-\beta)}\right\}\right)
$$

and its solutions in two classes of functions, namely

$$
\begin{aligned}
& \mathcal{I}=\left\{\varphi: \mathbb{R} \rightarrow \mathbb{R} \mid \varphi \text { is increasing, }\left.\varphi\right|_{(-\infty, 0]}=0,\left.\varphi\right|_{[1, \infty)}=1\right\}, \\
& \mathcal{C}=\left\{\varphi: \mathbb{R} \rightarrow \mathbb{R} \mid \varphi \text { is continuous, }\left.\varphi\right|_{(-\infty, 0]}=0,\left.\varphi\right|_{[1, \infty)}=1\right\} .
\end{aligned}
$$

We prove that the above equation has at most one solution in $\mathcal{C}$ and that for some parameters $\alpha, \beta$ and $p$ such a solution exists, and for some it does not. We also determine all solutions of the equation in $\mathcal{I}$ and we show the exact connection between solutions in both classes.

1. Introduction. In [4] M. Corsolini considered solutions $\psi:[0,1] \rightarrow$ $[0,1]$ of the functional equation

$$
\psi(x)= \begin{cases}p \psi\left[f_{s}(x)\right]+q \psi\left[f_{v}(x)\right] & \text { if } f_{v}(x) \in[0,1), \\ p \psi\left[f_{s}(x)\right]+q & \text { if } f_{v}(x) \in[1, \infty),\end{cases}
$$

with given numbers $\alpha, \beta, p, q \in(0,1)$ such that $p+q=1$ and functions $f_{s}:[0,1] \rightarrow[0,1], f_{v}:[0,1] \rightarrow\left[0, \max \left\{1, \beta \alpha^{-1}\right\}\right]$ defined by

$$
f_{s}(x)= \begin{cases}0 & \text { if } x \in[0, \beta] \\ \frac{x-\beta}{1-\beta} & \text { if } x \in(\beta, 1]\end{cases}
$$

2000 Mathematics Subject Classification: Primary 39B22, 39B12; Secondary 60E05, $26 \mathrm{~A} 30$.

Key words and phrases: functional equations, increasing solutions, continuous distribution functions.

This research was supported by the Silesian University Mathematics Department (Functional Equations in a Single Variable program). 


$$
f_{v}(x)= \begin{cases}\frac{x}{\alpha} & \text { if } x \in[0, \beta] \\ \frac{x(\alpha-\beta)+\beta(1-\alpha)}{\alpha(1-\beta)} & \text { if } x \in(\beta, 1] .\end{cases}
$$

For more details see [3] where the connection of this problem with a problem from game theory can be found. In a private correspondence M. Corsolini asked about the existence of monotonic solutions $\psi$ of $(1)$ such that $\psi(0)=0$ and $\psi(1)=1$.

The following result gives a positive answer to this question in the case where $\alpha \leq \beta$ (see [10] and [11]).

TheOREM A. If $\alpha \leq \beta$, then equation (1) has exactly one bounded solution $\psi:[0,1] \rightarrow \mathbb{R}$ such that $\psi(0)=0$ and $\psi(1)=1$. Moreover:

(i) $\psi$ is continuous and increasing.

(ii) If $\alpha=\beta$, then $\psi$ is strictly increasing and either absolutely continuous or singular.

(iii) If $\alpha<\beta$ then there exists a family $\mathcal{J}$ of disjoint open subintervals of $(0,1)$ such that $\psi$ is constant on each of them and $[0,1] \backslash \bigcup \mathcal{J}$ is of Lebesgue measure zero.

In this paper we are interested in the case where $\beta<\alpha$.

Assume $\beta<\alpha$ and define functions $f_{1}, f_{2}: \mathbb{R} \rightarrow \mathbb{R}$ by

$$
f_{1}(x)=\frac{x-\beta}{1-\beta} \quad \text { and } f_{2}(x)= \begin{cases}\frac{x}{\alpha} & \text { if } x \leq \beta, \\ \frac{x(\alpha-\beta)+\beta(1-\alpha)}{\alpha(1-\beta)} & \text { if } x>\beta .\end{cases}
$$

It is obvious that $f_{1}$ and $f_{2}$ are continuous, strictly increasing,

$$
f_{1}(x)<x<f_{2}(x)<1 \quad \text { for every } x \in(0,1)
$$

and

$$
f_{1}(x) \leq 0 \quad \text { for every } x \in(-\infty, \beta]
$$

Now, the question of $\mathrm{M}$. Corsolini can be restated as the question of existence of an increasing solution $\varphi: \mathbb{R} \rightarrow \mathbb{R}$ of the equation

$$
\varphi(x)=p \varphi\left[f_{1}(x)\right]+q \varphi\left[f_{2}(x)\right]
$$

such that

$$
\left.\varphi\right|_{(-\infty, 0]}=0 \text { and }\left.\varphi\right|_{[1, \infty)}=1 .
$$

We first observe that the answer to the question of M. Corsolini is positive. More precisely, the function $\chi_{[1, \infty)}$ is a solution of equation (E) satisfying condition (4). (Here and throughout, $\chi_{I}$ denotes the characteristic function, defined on the real line, of the set $I$.) 
Since we have the existence of a solution of (E) satisfying (4) we can ask about its uniqueness. The next observation suggests that equation (E) may have a lot of solutions $\varphi: \mathbb{R} \rightarrow \mathbb{R}$ satisfying (4).

REMARK 1. If $\varphi: \mathbb{R} \rightarrow \mathbb{R}$ is a solution of equation (E) satisfying condition (4), then for every $\lambda \in \mathbb{R}$ the function $\phi: \mathbb{R} \rightarrow \mathbb{R}$ defined by

$$
\phi(x)= \begin{cases}\lambda \varphi(x) & \text { if } x \in(-\infty, 1) \\ 1 & \text { if } x \in[1, \infty)\end{cases}
$$

is a solution of $(\mathrm{E})$ satisfying $\left.\phi\right|_{(-\infty, 0]}=0$ and $\left.\phi\right|_{[1, \infty)}=1$.

2. An example. To show that equation (E) may have many solutions $\varphi: \mathbb{R} \rightarrow \mathbb{R}$ satisfying (4) and, moreover, that the case $\beta<\alpha$ is different from that studied in [10] and [11] we consider the following situation.

ExAmple. Fix $0<\beta<\alpha<1$ and let $\sim$ be the equivalence relation on $\mathbb{R}$ defined by

$$
x \sim y \Leftrightarrow \bigvee_{n \in \mathbb{N}} \bigvee_{g_{1}, \ldots, g_{n} \in\left\{f_{1}, f_{2}, f_{1}^{-1}, f_{2}^{-1}\right\}}\left(x=g_{1} \circ \cdots \circ g_{n}(y)\right) .
$$

Equivalence relations of this type appear in a natural manner (see e.g. [2] or $[7])$. Let $[x]$ denote the equivalence class of $x$ and let $M$ denote a complete set of representatives of all equivalence classes of the relation $\sim$.

Fix a function $\lambda: M \rightarrow \mathbb{R}$ and define $\phi: \mathbb{R} \rightarrow \mathbb{R}$ by

$$
\phi(x)= \begin{cases}0 & \text { if } x \in(-\infty, 0] \\ \lambda(y) x & \text { if } x \in[y] \cap(0,1) \text { and } y \in M \\ 1 & \text { if } x \in[1, \infty) .\end{cases}
$$

Simple calculations show that

$$
\phi(x)=(1-\alpha) \phi\left[f_{1}(x)\right]+\alpha \phi\left[f_{2}(x)\right]
$$

for every $x \in \mathbb{R}$. In particular, for every $\lambda \in[0,1]$ the function $\varphi: \mathbb{R} \rightarrow \mathbb{R}$ defined by

$$
\varphi(x)= \begin{cases}0 & \text { if } x \in(-\infty, 0] \\ \lambda x & \text { if } x \in(0,1) \\ 1 & \text { if } x \in[1, \infty)\end{cases}
$$

is an increasing solution of (5) satisfying (4).

In what follows we are interested in solutions $\varphi$ of $(\mathrm{E})$ in the following two classes of functions:

$$
\begin{aligned}
& \mathcal{I}=\left\{\varphi: \mathbb{R} \rightarrow \mathbb{R} \mid \varphi \text { is increasing, }\left.\varphi\right|_{(-\infty, 0]}=0,\left.\varphi\right|_{[1, \infty)}=1\right\} \\
& \mathcal{C}=\left\{\varphi: \mathbb{R} \rightarrow \mathbb{R} \mid \varphi \text { is continuous, }\left.\varphi\right|_{(-\infty, 0]}=0,\left.\varphi\right|_{[1, \infty)}=1\right\}
\end{aligned}
$$


3. The uniqueness of solutions of $(\mathrm{E})$ in the class $\mathcal{C}$. On account of the Example we see that equation (E) may have a lot of solutions in the class $\mathcal{I}$. The first of our results shows that in $\mathcal{C}$ the situation is different.

TheOREM 1. Equation (E) has at most one solution in the class $\mathcal{C}$.

Proof. Let $\varphi_{1}, \varphi_{2} \in \mathcal{C}$ be solutions of (E) and put $\phi=\varphi_{1}-\varphi_{2}$. Then $\phi$ is a continuous solution of $(\mathrm{E})$ vanishing on $(-\infty, 0] \cup[1, \infty)$. Put

$$
M=\sup \{|\phi(x)|: x \in \mathbb{R}\}
$$

and suppose, contrary to our claim, that $M>0$. Let

$$
x_{0}=\inf \{x \in(0,1):|\phi(x)|=M\} \in(0,1) .
$$

By (2), $f_{1}\left(x_{0}\right)<x_{0}$. Then $\left|\phi\left[f_{1}\left(x_{0}\right)\right]\right|<M$, whence

$$
M=\left|\phi\left(x_{0}\right)\right| \leq p\left|\phi\left[f_{1}\left(x_{0}\right)\right]\right|+q\left|\phi\left[f_{2}\left(x_{0}\right)\right]\right|<p M+q M=M,
$$

a contradiction.

4. Some properties of solutions of $(\mathrm{E})$ in the classes $\mathcal{I}$ and $\mathcal{C}$. To get information about the existence of a solution of $(\mathrm{E})$ in the class $\mathcal{C}$ we need some properties of solutions of $(\mathrm{E})$ in $\mathcal{I}$ and $\mathcal{C}$.

Lemma 1. If $\varphi \in \mathcal{I}$ is a solution of $(\mathrm{E})$, then $\varphi$ is continuous at every point $x \neq 1$.

Proof. The function $\varphi_{0}: \mathbb{R} \rightarrow \mathbb{R}$ given by

$$
\varphi_{0}(x)=\lim _{y \rightarrow x^{+}} \varphi(y)-\lim _{z \rightarrow x^{-}} \varphi(z)
$$

is a nonnegative solution of $(\mathrm{E})$ such that

$$
\sum_{j=0}^{n-1} \varphi_{0}\left(x_{j}\right) \leq 1 \quad \text { whenever } 0 \leq x_{0}<\cdots<x_{n-1} \leq 1,
$$

and $\varphi_{0}(x)=0$ if and only if $\varphi$ is continuous at $x$. It is enough to show that $\varphi_{0}$ vanishes on $[0,1)$.

Since $\varphi_{0}(0)=q \varphi_{0}(0)$, we have $\varphi_{0}(0)=0$. Suppose

$$
L:=\sup \left\{\varphi_{0}(x): x \in(0,1)\right\}>0,
$$

fix a positive integer $n \geq 1 / L+q / p$ and an $x_{0} \in(0,1)$ such that

$$
\varphi_{0}\left(x_{0}\right)>\left(1-q^{n}\right) L \text {. }
$$

Then

$$
\left(1-q^{n}\right) L<\varphi_{0}\left(x_{0}\right)=p \varphi_{0}\left[f_{1}\left(x_{0}\right)\right]+q \varphi_{0}\left[f_{2}\left(x_{0}\right)\right] \leq p L+q \varphi_{0}\left[f_{2}\left(x_{0}\right)\right],
$$

whence

$$
\varphi_{0}\left(x_{1}\right)>\left(1-q^{n-1}\right) L,
$$

where $x_{1}:=f_{2}\left(x_{0}\right)$. By $(2), x_{1} \in\left(x_{0}, 1\right)$. By induction we obtain 
$\varphi_{0}\left(x_{j}\right)>\left(1-q^{n-j}\right) L, \quad$ where $\quad x_{j}=f_{2}^{j}\left(x_{0}\right)$ for $j=0,1, \ldots, n-1$, and $\left(x_{0}, x_{1}, \ldots, x_{n-1}\right)$ is a strictly increasing sequence of numbers from $(0,1)$. Consequently,

$$
\sum_{j=0}^{n-1} \varphi_{0}\left(x_{j}\right)>L\left(n-\sum_{j=0}^{n-1} q^{n-j}\right)>L\left(n-\frac{q}{1-q}\right) \geq 1
$$

which contradicts (6).

From now on let $\left(\varphi_{n}: n \in \mathbb{N}\right)$ denote a sequence of functions from $\mathbb{R}$ to $\mathbb{R}$ defined as follows:

$$
\varphi_{1}(x)=\chi_{(0, \infty)}(x) \quad \text { and } \quad \varphi_{n+1}(x)=p \varphi_{n}\left[f_{1}(x)\right]+q \varphi_{n}\left[f_{2}(x)\right]
$$

for any $n \in \mathbb{N}$ and $x \in \mathbb{R}$.

By induction we get the following observation.

LEMma 2. The sequence $\left(\varphi_{n}: n \in \mathbb{N}\right)$ defined by $(7)$ is a decreasing sequence of functions from $\mathcal{I}$, and its limit $\Phi$,

$$
\Phi(x)=\lim _{n \rightarrow \infty} \varphi_{n}(x) \quad \text { for every } x \in \mathbb{R},
$$

is a solution of $(\mathrm{E})$ and belongs to $\mathcal{I}$.

Proof. Since

$$
\varphi_{2}(x)=p \varphi_{1}\left[f_{1}(x)\right]+q \varphi_{1}\left[f_{2}(x)\right]=p \chi_{(\beta, \infty)}(x)+q \chi_{(0, \infty)}(x) \leq \varphi_{1}(x)
$$

for every $x \in \mathbb{R}$, the obvious induction shows that $\left(\varphi_{n}: n \in \mathbb{N}\right)$ decreases. The rest is evident.

THEOREM 2. Equation (E) has a solution in the class $\mathcal{C}$ if and only if the function $\Phi$ defined by (8) and (7) is continuous.

Proof. If $\Phi$ is continuous, then, by Lemma 2, it is a solution of $(\mathrm{E})$ in the class $\mathcal{C}$. Assume now that $\psi \in \mathcal{C}$ is a solution of $(\mathrm{E})$. Let $M=\sup \{\psi(x)$ : $x \in \mathbb{R}\}$. Obviously, $M \in[1, \infty)$. Moreover, there exists a $y \in[0,1]$ such that $M=\psi(y)$. By Remark 1, the function $\Psi: \mathbb{R} \rightarrow \mathbb{R}$ given by

$$
\Psi(x)= \begin{cases}M^{-1} \psi(x) & \text { if } x \in(-\infty, 1) \\ 1 & \text { if } x \in[1, \infty)\end{cases}
$$

is a solution of $(\mathrm{E})$. Since $\Psi \leq \varphi_{1}$, an obvious induction shows that $\Psi \leq \varphi_{n}$ for every $n \in \mathbb{N}$. Consequently, $\Psi \leq \Phi$. In particular,

$$
M=\psi(y)=\lim _{x \rightarrow y^{-}} \psi(x)=M \lim _{x \rightarrow y^{-}} \Psi(x) \leq M \lim _{x \rightarrow y^{-}} \Phi(x) .
$$

From this and Lemma 2 we see that

$$
1 \leq \lim _{x \rightarrow y^{-}} \Phi(x) \leq \lim _{x \rightarrow 1^{-}} \Phi(x) \leq \Phi(1)=1
$$

which together with Lemma 1 gives $\Phi \in \mathcal{C}$. 
Lemma 3. If $\Phi$ is continuous, then it maps $(0,1)$ into itself.

Proof. Suppose that there exists an $x<1$ such that $\Phi(x)=1$. Then from Lemma 2 we deduce that there exists a $y \in(0,1)$ such that $\Phi(x)<1$ for every $x<y$ and $\Phi(x)=1$ for every $x \geq y$. This together with (2) gives

$$
1=\Phi(y)=p \Phi\left[f_{1}(y)\right]+q \Phi\left[f_{2}(y)\right]<p+q=1,
$$

a contradiction.

Now suppose that there exists an $x>0$ such that $\Phi(x)=0$. Then from Lemma 2 we deduce that there exists a $y \in(0,1)$ such that $\Phi(x)=0$ for every $x \leq y$ and $\Phi(x)>0$ for every $x>y$. This together with (2) gives

$$
0=\Phi(y)=p \Phi\left[f_{1}(y)\right]+q \Phi\left[f_{2}(y)\right]=q \Phi\left[f_{2}(y)\right]>0,
$$

a contradiction.

Lemma 4. If $\varphi \in \mathcal{I}$ is a solution of equation (E) which is constant on an interval $I \subset \mathbb{R}$, then $\varphi$ is also constant on the intervals $f_{1}(I)$ and $f_{2}(I)$.

Proof. Fix $x_{1}, x_{2} \in I$ such that $x_{1}<x_{2}$. Then

$$
p \varphi\left[f_{1}\left(x_{1}\right)\right]+q \varphi\left[f_{2}\left(x_{1}\right)\right]=\varphi\left(x_{1}\right)=\varphi\left(x_{2}\right)=p \varphi\left[f_{1}\left(x_{2}\right)\right]+q \varphi\left[f_{2}\left(x_{2}\right)\right]
$$

and since all the functions occurring above are increasing we have

$$
\varphi\left[f_{1}\left(x_{1}\right)\right]=\varphi\left[f_{1}\left(x_{2}\right)\right] \quad \text { and } \quad \varphi\left[f_{2}\left(x_{1}\right)\right]=\varphi\left[f_{2}\left(x_{2}\right)\right] .
$$

This proves the assertion.

THEOREM 3. If $\Phi$ is continuous, then it is strictly increasing on the interval $[0,1]$ and it is either absolutely continuous or singular.

Proof. Suppose that $\Phi$ is not strictly increasing on $[0,1]$ and let $[a, b] \subset$ $[0,1]$ be an interval of maximal length on which $\Phi$ is constant. It follows from Lemma 3 that $[a, b] \subset(0,1)$. Using Lemma 4 we see that $\phi$ is constant on the intervals $\left[f_{1}(a), f_{1}(b)\right]$ and $\left[f_{2}(a), f_{2}(b)\right]$. Since $f_{1}(b)-f_{1}(a)>b-a$, it follows that $\left[f_{1}(a), f_{1}(b)\right] \cap(0,1)=\emptyset$, which together with $(2)$ and $(3)$ gives $b \leq \beta$. Hence $f_{2}(b)-f_{2}(a)>b-a$ and thus $\left[f_{2}(a), f_{2}(b)\right] \cap(0,1)=\emptyset$; this contradicts the fact that $f_{2}([0,1]) \subset[0,1]$.

Now we show that the unique solution of $(\mathrm{E})$ in the class $\mathcal{C}$ is either absolutely continuous or singular. Let $\Phi \in \mathcal{C}$ be the unique solution of $(\mathrm{E})$. By the Canonical Lebesgue Decomposition Theorem (see, e.g., [9, Theorem 7.4.9]) there exist exactly one absolutely continuous (and increasing) function $\varphi_{\mathrm{a}}:[0,1] \rightarrow \mathbb{R}$ and exactly one singular (continuous and increasing) function $\varphi_{\mathrm{s}}:[0,1] \rightarrow \mathbb{R}$ such that $\varphi_{\mathrm{a}}(0)=0$ and

$$
\Phi(x)=\varphi_{\mathrm{a}}(x)+\varphi_{\mathrm{s}}(x)
$$

for every $x \in[0,1]$. 
Assume that $\varphi_{\mathrm{a}}$ does not vanish. We shall show that $\Phi$ is absolutely continuous. Let $c=1 / \varphi_{\mathrm{a}}(1)$ and define $\phi_{\mathrm{a}}: \mathbb{R} \rightarrow \mathbb{R}$ and $\phi_{\mathrm{s}}: \mathbb{R} \rightarrow \mathbb{R}$ by

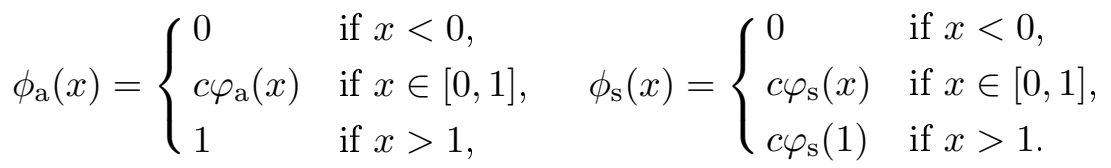

Observe that the functions $\phi_{\mathrm{a}}$ and $\phi_{\mathrm{s}}$ so defined are continuous, increasing,

$$
c \Phi=\phi_{\mathrm{a}}+\phi_{\mathrm{s}}
$$

and

$$
\begin{aligned}
\phi_{\mathrm{a}}(x)+\phi_{\mathrm{s}}(x) & =c \Phi(x)=c\left[p \Phi\left[f_{1}(x)\right]+q \Phi\left[f_{2}(x)\right]\right] \\
& =p \phi_{\mathrm{a}}\left[f_{1}(x)\right]+q \phi_{\mathrm{a}}\left[f_{2}(x)\right]+p \phi_{\mathrm{s}}\left[f_{1}(x)\right]+q \phi_{\mathrm{s}}\left[f_{2}(x)\right]
\end{aligned}
$$

for every $x \in \mathbb{R}$. Since $\phi_{\mathrm{a}} \circ f_{1}$ and $\phi_{\mathrm{a}} \circ f_{2}$ are absolutely continuous, and $\phi_{\mathrm{s}} \circ f_{1}$ and $\phi_{\mathrm{s}} \circ f_{2}$ are singular, the uniqueness of the decomposition implies that there exists a real constant $d$ such that

$$
\phi_{\mathrm{a}}(x)=p \phi_{\mathrm{a}}\left[f_{1}(x)\right]+q \phi_{\mathrm{a}}\left[f_{2}(x)\right]+d
$$

for every $x \in \mathbb{R}$. Thus

$$
1=\phi_{\mathrm{a}}(1)=p \phi_{\mathrm{a}}\left[f_{1}(1)\right]+q \phi_{\mathrm{a}}\left[f_{2}(1)\right]+d=1+d,
$$

so $d=0$, and hence $\phi_{\mathrm{a}} \in \mathcal{C}$. By Theorem 1 we get $\phi_{\mathrm{a}}=\Phi$.

5. The existence of solutions of $(\mathbf{E})$ in the class $\mathcal{C}$. We begin with the case where $q \geq \alpha$.

Lemma 5. Assume that $q \geq \alpha$. Then

$$
\Phi(x) \geq x \quad \text { for every } x \in[0,1] .
$$

Proof. It is enough to prove (by induction) that $\varphi_{n}(x) \geq x$ for all $n \in \mathbb{N}$ and $x \in[0,1]$.

THEOREM 4. If $q \geq \alpha$, then equation (E) has exactly one solution in the class $\mathcal{C}$. Moreover, this solution is strictly increasing on $[0,1]$ and either absolutely continuous or singular.

Proof. By Lemmas 2, 1 and 5, and by Theorem 2, we get the existence. The uniqueness follows from Theorem 1 . The remaining assertion is a consequence of Theorem 3.

THEOREM 5. Assume that

$$
q \leq \alpha-p \beta
$$

Then equation $(\mathrm{E})$ has no solution in the class $\mathcal{C}$. 
Proof. Assumption (10) is equivalent to the inequality

$$
p(1-\beta)+q \alpha \frac{1-\beta}{\alpha-\beta} \leq 1 .
$$

Suppose that, contrary to our claim, $\varphi \in \mathcal{C}$ is a solution of (E). Then by Lemma 3 we have

$$
\int_{0}^{\beta / \alpha} \varphi(x) d x>0
$$

and

$$
\begin{aligned}
\int_{0}^{1} \varphi(x) d x= & q \int_{0}^{\beta} \varphi\left(\frac{x}{\alpha}\right) d x+p \int_{\beta}^{1} \varphi\left(\frac{x-\beta}{1-\beta}\right) d x \\
& +q \int_{\beta}^{1} \varphi\left(\frac{x(\alpha-\beta)+\beta(1-\alpha)}{\alpha(1-\beta)}\right) d x \\
= & q \alpha \int_{0}^{\beta / \alpha} \varphi(y) d y+p(1-\beta) \int_{0}^{1} \varphi(y) d y \\
& +q \alpha \frac{1-\beta}{\alpha-\beta} \int_{\beta / \alpha}^{1} \varphi(y) d y \\
< & {\left[p(1-\beta)+q \alpha \frac{1-\beta}{\alpha-\beta}\right] \int_{0}^{1} \varphi(y) d y \leq \int_{0}^{1} \varphi(y) d y }
\end{aligned}
$$

a contradiction.

6. Solutions of $(\mathbf{E})$ in the class $\mathcal{I}$. We begin with a general result connecting the existence of a solution of $(\mathrm{E})$ in the class $\mathcal{C}$ with the set of all solutions of $(\mathrm{E})$ in the class $\mathcal{I}$.

THEOREM 6. (i) Equation (E) has a solution in the class $\mathcal{C}$ if and only if $(\mathrm{E})$ has a solution $\psi \in \mathcal{I}$ such that $\psi \neq \chi_{[1, \infty)}$. Moreover:

(ii) If $\varphi \in \mathcal{C}$ is a solution of (E), then $\psi \in \mathcal{I}$ is a solution of (E) if and only if there exists a $\lambda \in[0,1]$ such that

$$
\psi(x)= \begin{cases}\lambda \varphi(x) & \text { if } x \in(-\infty, 1), \\ 1 & \text { if } x \in[1, \infty) .\end{cases}
$$

(iii) If $\psi \in \mathcal{I}, \psi \neq \chi_{[1, \infty)}$, is a solution of (E), then there exists a $\gamma \in[1, \infty)$ such that the function $\varphi: \mathbb{R} \rightarrow \mathbb{R}$ given by

$$
\varphi(x)= \begin{cases}\gamma \psi(x) & \text { if } x \in(-\infty, 1), \\ 1 & \text { if } x \in[1, \infty)\end{cases}
$$

is a solution of $(\mathrm{E})$ in the class $\mathcal{C}$. 
Proof. If $\varphi \in \mathcal{C}$ is a solution of (E), then by Remark 1 , so is $\psi$ defined by (11). Since $\lambda \in[0,1]$, by Theorem 3 we get $\psi \in \mathcal{I}$.

Assume now that $\psi \in \mathcal{I}$ is a solution of (E). From Lemma 1 we see that $\psi$ is continuous at every point $x \neq 1$.

If $\lim _{x \rightarrow 1^{-}} \psi(x)=0$, then $\psi=\chi_{[1, \infty)}$. Hence (11) holds with $\lambda=0$.

If $\lim _{x \rightarrow 1^{-}} \psi(x) \in(0,1]$, then the function $\varphi: \mathbb{R} \rightarrow \mathbb{R}$ defined by (12) with

$$
\gamma=\frac{1}{\lim _{x \rightarrow 1^{-}} \psi(x)}
$$

is a solution of (E) (cf. Remark 1) continuous at 1 and $\varphi \in \mathcal{I}$. This, together with Lemma 1 , shows that $\varphi \in \mathcal{C}$.

From Theorems 4, 5 and 7 we get the following two corollaries.

Corollary 1. Assume that $q \geq \alpha$ and let $\varphi \in \mathcal{C}$ be the unique solution of $(\mathrm{E})$. Then every solution $\psi \in \mathcal{I}$ of $(\mathrm{E})$ is of the form (11) with some $\lambda \in[0,1]$.

COROLlary 2. If (10) holds, then $\chi_{[1, \infty)}$ is the only solution of $(\mathrm{E})$ in the class $\mathcal{I}$.

7. Consequences of a theorem of K. Baron. We first observe that equation $(\mathrm{E})$ can be rewritten in the form

$$
\varphi(x)=\int_{\Omega} \varphi(\tau(x, \omega)) d P(\omega),
$$

where $\Omega=\{1,2\}$ and $P$ is a probability measure on $2^{\Omega}$ given by $P(\{1\})=p$, $P(\{2\})=q$ and $\tau(\cdot, \omega)=f_{\omega}$ for $\omega \in\{1,2\}$. Now we can to try use known results on equation (13) in a much more general setting to get information on solutions of $(\mathrm{E})$ in the class $\mathcal{I}$ (or equivalently, by Theorem 6 , in the class $\mathcal{C}$ ). To the best of our knowledge the following theorem of K. Baron [1] is the most general result applicable to equation (E).

Theorem B (K. Baron). Assume that $(\Omega, \mathcal{A}, P)$ is a probability space and that $\tau: \mathbb{R} \times \Omega \rightarrow \mathbb{R}$ is a function such that for every $\omega \in \Omega$ the function $\tau(\cdot, \omega)$ is strictly increasing and transforms $\mathbb{R}$ onto $\mathbb{R}$, and for every $x \in \mathbb{R}$ the function $\tau(x, \cdot)$ is a random variable. Let $L: \Omega \rightarrow(0, \infty)$ be a random variable such that

$$
|\tau(x, \omega)-\tau(y, \omega)| \geq L(\omega)|x-y| \quad \text { for all } x, y \in \mathbb{R}, \omega \in \Omega
$$

and

$$
0<\int_{\Omega} \log L(\omega) d P(\omega)<\infty
$$


If there exists an $x_{0} \in \mathbb{R}$ such that

$$
\int_{\left\{\omega \in \Omega:\left|\tau\left(x_{0}, \omega\right)-x_{0}\right|>L(\omega)\right\}} \log \frac{\left|\tau\left(x_{0}, \omega\right)-x_{0}\right|}{L(\omega)} d P(\omega)<\infty,
$$

then equation (13) has exactly one solution in the class of probability distribution functions.

We wish to apply Theorem B to equation (E). Observe that since both $f_{1}$ and $f_{2}$ map $\mathbb{R}$ onto $\mathbb{R}$, it follows that the main assumptions on $\tau$ hold. It is evident that condition (16) holds with any $x_{0} \in \mathbb{R}$. Moreover, elementary calculations shows that (14) holds with $L$ given by

$$
L(1)=\frac{1}{1-\beta}, \quad L(2)=\frac{\alpha-\beta}{\alpha(1-\beta)}
$$

and this function $L$ is the best possible. Consequently, condition (15) now reads

$$
0<p \log \frac{1}{1-\beta}+q \log \frac{\alpha-\beta}{\alpha(1-\beta)}
$$

or equivalently

$$
\frac{\beta}{1-(1-\beta)^{1 / q}}<\alpha
$$

Let us mention here that condition (15) has been used in some papers on functional equations (see e.g. [5] or [8]) and on iterated function systems (see e.g. [6] and the references therein).

As an immediate consequence of Theorem B and Theorem 6 we get the following result.

Theorem 7. Assume (17). Then:

(i) Equation (E) has no solution in the class $\mathcal{C}$.

(ii) The function $\chi_{[1, \infty)}$ is the unique solution of $(\mathrm{E})$ in the class $\mathcal{I}$.

We know that in some cases condition (17) is stronger than condition (10); e.g. in the case where $p=q=1 / 2$ condition (17) can be written as $1+$ $\alpha \beta<2 \alpha$, whereas condition (10) takes the form $1+\beta \leq 2 \alpha$. Unfortunately, we do not know if such a connection is valid for all parameters $p, \alpha$ and $\beta$ such that $\max \{\beta, q\}<\alpha$.

We end this paper by asking when equation (E) has a solution in the class $\mathcal{C}$ if $q<\alpha$ and neither (17) nor (10) holds.

Acknowledgements. The main part of this work was done while the first author was visiting the Karl Franzens Universität in Graz. He thanks the Karl Franzens Universität and especially Professor D. Gronau for hospitality. 


\section{References}

[1] K. Baron, Solutions of iterative functional equations resulting from iterated function systems theory, manuscript.

[2] L. Bartłomiejczyk, Solutions with big graph of homogeneous functional equations in a single variable, Aequationes Math. 56 (1998), 149-156.

[3] M. Corsolini, Numeri normali, indipendenza statistica e sistemi moltiplicativi ortogonali nelle basi di numerazione non uniformi,

http://www.liberliber.it/biblioteca/tesi/scienze_matematiche_fisiche_e_naturali/.

[4] - Dello scommettere su monete truccate, manuscript.

[5] G. Derfel, Probabilistic method for a class of functional-differential equations, Ukrain. Mat. Zh. 41 (1989), 1322-1327 (in Russian); English transl.: Ukrainian Math. J. 41 (1989), 1137-1141.

[6] P. Diaconis and D. Freedman, Iterated random functions, SIAM Rev. 41 (1999), $45-76$.

[7] G. L. Forti and L. Paganoni, An iterative method for solving a system of functional equations, Adv. Math. 105 (1994), 111-161.

[8] R. Kapica, Sequences of iterates of random-valued vector functions and continuous solutions of a linear functional equation of infinite order, Bull. Polish Acad. Sci. Math. 50 (2002), 447-455.

[9] S. Łojasiewicz, An Introduction to the Theory of Real Functions, Wiley, Chichester, 1988.

[10] J. Morawiec, On a linear functional equation, Bull. Polish Acad. Sci. Math. 43 (1995), 131-142.

[11] —, Some properties of probability distribution solutions of linear functional equations, Aequationes Math. 56 (1998), 81-90.

Janusz Morawiec

Institute of Mathematics

Silesian University

Bankowa 14

PL-40-007 Katowice, Poland

E-mail: morawiec@ux2.math.us.edu.pl
Ludwig Reich Institut für Mathematik Karl Franzens Universität Heinrichstrasse 36 A-8010 Graz, Austria E-mail: ludwig.reich@kfunigraz.ac.at

Received November 9, 2005;

received in final form February 14, 2006 